\title{
278.
}

\section{NOTE ON THE SINGULAR SOLUTIONS OF DIFFERENTIAL EQUATIONS.}

[From the Quarterly Journal of Pure and Applied Mathematics, vol. III. (1860), pp. 36, 37.]

THE following investigation (which has been in my possession for a good many years) affords I think a simple explanation of the theory of the singular solutions of differential equations.

Let the primitive equation be

$$
c^{n}+P c^{n-1}+Q c^{n-2}+\ldots=0,
$$

where $c$ is the arbitrary constant and $P, Q \ldots$ are any functions of $x, y$; then the differential equation is obtained by eliminating $c$ from the foregoing equation and the derived equation

$$
P^{\prime} c^{n-1}+Q^{\prime} c^{n-2}+\ldots=0
$$

and the result may be represented by

Assume now

$$
F\left(P, Q, \ldots, P^{\prime}, Q^{\prime}, \ldots\right)=0 .
$$

then we have

$$
c^{n}+P c^{n-1}+Q c^{n-2}+\ldots=(c+X)(c+Y)(c+Z) \ldots
$$

$$
\begin{aligned}
& P=X+Y+Z+\& c . \\
& Q=X Y+X Z+Y Z+\& c . \\
& \& c .
\end{aligned}
$$

and consequently

$$
\begin{aligned}
& P^{\prime}=\dot{X}^{\prime}+Y^{\prime}+Z^{\prime}+\& c . \\
& Q^{\prime}=(Y+Z+\& c .) X^{\prime}+\& c . \\
& \& c .,
\end{aligned}
$$


and substituting these values in the function $F\left(P, Q, \ldots P^{\prime}, Q^{\prime}, \ldots\right)$ it is clear that we shall have $F\left(P, Q, \ldots, P^{\prime}, Q^{\prime}, \ldots\right)=U X^{\prime} Y^{\prime} Z^{\prime} \ldots$ where $U$ is a symmetrical function of $X, Y, Z, \& c$., and therefore a function of $P, Q, \ldots ;$ and this equation will be identically true whatever values we attribute to $X^{\prime}, Y^{\prime}, Z^{\prime}, \ldots$, hence putting these quantities respectively equal to unity, we have

$$
\begin{aligned}
& P^{\prime}=n, \\
& Q^{\prime}=(n-1) P, \\
& R^{\prime}=(n-2) Q, \\
& \text { \&c. }
\end{aligned}
$$

and with these values

$$
U=F\left(P, Q, \ldots, P^{\prime}, Q^{\prime}, \ldots\right),
$$

that is $U=0$ is the result obtained by eliminating $c$ from the primitive equation and the equation

$$
n c^{n-1}+(n-1) P c^{n-2}+\ldots=0,
$$

which is the equation obtained by differentiating the primitive equation with respect to the arbitrary constant $c$ : that is, $U=0$ being the singular solution, the differential equation is

$$
U X^{\prime} Y^{\prime} Z^{\prime} \ldots=0 .
$$

It is to be remarked that ( $P, Q, \& c$. being rational and integral functions) then if the roots $X, Y, Z$, \&c. are also rational and integral functions, the differential equation contains $U$ as a separable rational and integral factor, but if the roots are irrational then the differential equation does not really contain the rational and integral factor $U$, but $X^{\prime} Y^{\prime} Z^{\prime} \ldots$ is here a rational fraction containing $U$ in the denominator and $U X^{\prime} Y^{\prime} Z^{\prime} \ldots$ is an indecomposable rational and integral function. This is easily verified à posteriori for a quadratic equation.

2, Stone Buildings, W.C., 28th January, 1858. 